\title{
Doppler Synthetic Aperture Hitchhiker Imaging
}

\author{
Ling Wang ${ }^{a}$, Can Evren Yarman $^{b}$ and Birsen Yazıc1 $^{c}$ \\ ${ }^{a}$ Department of Information and Communication Engineering, Nanjing University of \\ Aeronautics and Astronautics, Nanjing, Jiangsu 210016 China; \\ ${ }^{b}$ Houston Technology Center, WesternGeco Schlumberger, Houston, TX 77042 USA; \\ ${ }^{c}$ Department of Electrical, Computer and System Engineering, Rensselaer Polytechnic \\ Institute, Troy, NY 12180 USA
}

\begin{abstract}
We consider passive airborne receivers that use backscattered signals from sources of opportunity transmitting fixed-frequency waveforms, which we refer to as Doppler Synthetic Aperture Hitchhiker (DSAH). We present a novel image formation method for DSAH. Our method first correlates the windowed signal obtained from one receiver with the windowed, filtered, scaled and translated version of the received signal from another receiver, and then uses the microlocal analysis to reconstruct the scene radiance by the weighted-backprojection of the correlated signal. This imaging algorithm can put the visible edges of the scene radiance at the correct location, and under appropriate conditions, with correct strength. We show that the resolution of the image is directly related to the length of the support of the windowing function and the frequency of the transmitted waveform. We present numerical experiments to demonstrate the performance of the proposed method.
\end{abstract}

Keywords: Passive Radar, Imaging, Synthetic Aperture, Continuous Wave (CW), Doppler, Backprojection

\section{INTRODUCTION}

With the growing availability of transmitters of opportunity, such as radio, television and cell phone stations, there has been a growing interest in passive radar applications using sources of opportunity. ${ }^{1-13}$ Based on the fact that most of the transmitters of opportunity are single-frequency or ultra-narrowband sources, such as radio and TV stations, we consider passive airborne receivers that use backscattered signals from sources of opportunity transmitting fixed-frequency waveforms. These waveforms are also referred to as high-Doppler-resolution or continuous-wave $(\mathrm{CW})$ waveforms. Due to the passive synthetic aperture and the nature of the transmitted waveforms, we refer to the system under consideration as Doppler Synthetic Aperture Hitchhiker (DSAH).

We present a novel image formation method for DSAH. Our method first correlates the windowed signal obtained from one receiver with the windowed, filtered, scaled and translated version of the received signal from another receiver. This processing removes the transmitter related variables from the phase of the Fourier integral operator that relates the radiance of the scene and the correlated signal. We, next, use the microlocal analysis ${ }^{14-17}$ to reconstruct the scene radiance by the weighted-backprojection of the correlated signal. The analysis of the point spread function (PSF) of the imaging operator shows that this imaging algorithm can put the visible edges of the scene radiance at the correct location, and under appropriate conditions, with correct strength. We show that the resolution of the image is directly related to the length of the support of the windowing function and the frequency of the transmitted waveform. The final image of the scene radiance is formed by averaging over the images obtained for each pair of receivers at each time translation. We present numerical experiments to validate the theoretical analysis and demonstrate the performance of the proposed method.

Our passive imaging method has the following advantages: (1) as compared to the existing passive radar detection/imaging systems, ${ }^{1-12}$ it does not require receivers with high directives; (2) it can be used in the presence of both cooperative and non-cooperative sources of opportunity; (3) it can be used with stationary

Further author information: (Send correspondence to B.Y.)

B.Y.: E-mail: yazici@ecse.rpi.edu, Telephone: +1(518)276 2905, Fax: +1(518)276 6261

C.E.Y.: E-mail: yarman@ecse.rpi.edu

L.W.:E-mail: wanglrpi@gmail.com

Algorithms for Synthetic Aperture Radar Imagery XVII, edited by Edmund G. Zelnio, Frederick D. Garber, Proc. of SPIE Vol. 7699, 769903 - (c) 2010 SPIE · CCC code: 0277-786X/10/\$18 - doi: 10.1117/12.850023 


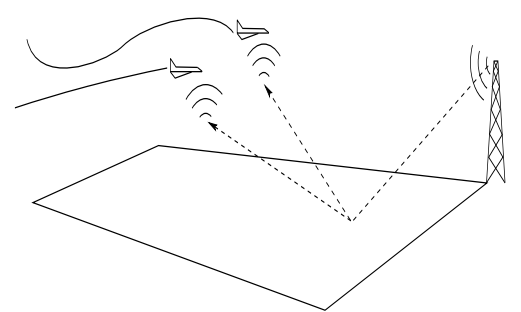

Figure 1: An illustration of the DSAH imaging geometry.

and/or mobile sources of opportunity; (4) it can be used with one or more airborne receivers. (5) it is an analytic reconstruction technique which can be made computationally efficient.

The organization of our paper is as follows: In Section 2, we first introduce the model of the received signal. Then we develop the forward model for DSAH and analyze the leading-order contributions to the windowed, filtered-scaled-and-correlated measurements. In Section 3, we develop a weighted-backprojection type image formation method for DSAH and analyze the underlying geometry and resolution of DSAH image formation. In Section 4, we present numerical simulations to verify the theoretical results and to demonstrate the performance of the DSAH image formation method. Finally, in Section 5 we conclude our discussion.

\section{DSAH MEASUREMENT MODEL}

\subsection{Model for the received signal}

We use the following notational conventions throughout the paper. The bold Roman, bold italic and Roman lower-case letters are used to denote variables in $\mathbb{R}^{3}, \mathbb{R}^{2}$ and $\mathbb{R}$, respectively, i.e., $\mathbf{z}=(\boldsymbol{z}, z) \in \mathbb{R}^{3}$, with $\boldsymbol{z} \in \mathbb{R}^{2}$ and $z \in \mathbb{R}$. The calligraphic letters $(\mathcal{F}, \mathcal{K}$ etc. $)$ are used to denote operators.

We make the assumption that the earth's surface is located at a position given by $\mathbf{z}=(\boldsymbol{z}, \boldsymbol{\psi}(\boldsymbol{z})) \in \mathbb{R}^{3}$, where $z \in \mathbb{R}^{2}$ and $\psi: \mathbb{R}^{2} \rightarrow \mathbb{R}$ is a known function for the ground topography. Furthermore, we assume that the scattering takes place in a thin region near the surface.

Under these assumptions, for a fixed-frequency waveform at frequency $\omega_{0}$, given a pair of transmitter and receiver antennas located at $\mathbf{T}$ and $\mathbf{R}$ respectively, we model the received signal by ${ }^{15}$

$$
f(t, \mathbf{R}, \mathbf{T}) \approx \int \frac{\mathrm{e}^{-\mathrm{i} \omega_{0}\left(t-(|\mathbf{R}-\mathbf{z}|+|\mathbf{z}-\mathbf{T}|) / c_{0}\right)}}{(4 \pi)^{2}|\mathbf{R}-\mathbf{z}||\mathbf{z}-\mathbf{T}|} \omega_{0}^{2} J_{\mathrm{tr}}\left(\omega_{0}, \widehat{\mathbf{z}-\mathbf{T}}, \mathbf{T}\right) J_{\mathrm{rc}}\left(\omega_{0}, \widehat{\mathbf{z}-\mathbf{R}}, \mathbf{R}\right) \rho(\boldsymbol{z}) d \boldsymbol{z}
$$

where $t$ denotes time, $c_{0}$ denotes the speed of light in free-space, $\rho(\boldsymbol{z})$ is the reflectivity function, and $J_{t r}$ and $J_{r c}$ are the transmitter and receiver antenna beam patterns, respectively. $\widehat{\mathbf{z}}=\mathbf{z} /|\mathbf{z}|$ denotes the unit vector in the direction of $\mathbf{z} \in \mathbb{R}^{3}$. For the rest of the paper, unless otherwise stated, we use $\mathbf{z}=\mathbf{z}(\boldsymbol{z})=(\boldsymbol{z}, \boldsymbol{\psi}(\boldsymbol{z}))$.

Our imaging method is applicable to both mobile and stationary sources of opportunity and also applicable to multiple transmitters and multiple transmit frequencies. However, for the rest of our discussion, we assume that there is a single, stationary transmitter of opportunity illuminating the scene. This allows us to simplify the analysis and distill the important aspects of our imaging theory.

Let $\mathbf{T} \in \mathbb{R}^{3}$ denote the location of the transmitter and let there be $N$ airborne receivers, each traversing a smooth trajectory $\gamma_{i}(t), i=1, \cdots, N$ as shown in Figure 1 . We define

$$
s_{i}(t)=f\left(t, \gamma_{i}(t), \mathbf{T}\right)
$$

as the received signal by the $i$ th receiver, $i=1,, \cdots, N$. 


\subsection{Windowed, filtered-scaled-and-translated correlation of received signals}

For a given point of interest $\boldsymbol{z}_{0}$ on the ground topography and a fixed $\tau^{\prime} \in \mathbb{R}$, we define the windowed, filteredscaled-and-translated correlation of the received signals $s_{i}$ and $s_{j}$ by

$$
c_{i j}^{z_{0}}\left(\tau^{\prime}, \tau, \mu\right)=\int \frac{s_{i}\left(t+\tau^{\prime}\right) s_{j}^{*}(\mu t+\tau)}{B_{i j}\left(z_{0}, t, \tau^{\prime}, \tau, \mu\right)} \phi(t)|t| d t,
$$

for some $\tau, \tau^{\prime} \in \mathbb{R}$ and $\mu \in \mathbb{R}^{+}, i, j=1, \cdots, N$, where $\phi(t)$ is a smooth compactly supported temporal windowing function centered at $t=0$; and $B_{i j}$ is a filter to be determined later; and * denotes the complex conjugation. To simplify our notation, we drop the superscripts $z_{0}$ from $c_{i j}^{z_{0}}\left(\tau^{\prime}, \tau, \mu\right)$ for the rest of our paper. Note that for a single receiver, we only have $c_{i j}=c_{11}$.

For non-cooperative sources of opportunity, we use a stochastic model for the transmitter antenna beam pattern, $J_{\mathrm{tr}}$, and the scene reflectivity, $\rho$. Let $C_{\rho}$ and $C_{J_{\mathrm{tr}}}$ denote the correlation function of $\rho$ and $J_{\mathrm{tr}}$, respectively,

$$
\begin{gathered}
C_{\rho}\left(\boldsymbol{z}, \boldsymbol{z}^{\prime}\right)=E\left[\rho(\boldsymbol{z}) \rho^{*}\left(\boldsymbol{z}^{\prime}\right)\right], \\
C_{J_{\mathrm{tr}}}\left(\omega_{0}, \boldsymbol{z}, \boldsymbol{z}^{\prime}, \mathbf{T}\right)=E\left[J_{\mathrm{tr}}\left(\omega_{0}, \widehat{\mathbf{z}-\mathbf{T}}, \mathbf{T}\right) J_{\mathrm{tr}}^{*}\left(\omega_{0}, \widehat{\mathbf{z}^{\prime}-\mathbf{T}}, \mathbf{T}\right)\right] .
\end{gathered}
$$

We make the assumption that the scene reflectivity $\rho$, and the transmit antenna beam pattern $J_{\text {tr }}$ are statistically independent. Furthermore, we make the incoherent-field approximation ${ }^{18}$ by assuming that $\rho$ and $J_{\text {tr }}$ satisfy the following equalities:

$$
\begin{aligned}
C_{\rho}\left(\boldsymbol{z}, \boldsymbol{z}^{\prime}\right) & =R_{\rho}(\boldsymbol{z}) \delta\left(\boldsymbol{z}-\boldsymbol{z}^{\prime}\right) \\
C_{J_{\mathrm{tr}}}\left(\omega_{0}, \boldsymbol{z}, \boldsymbol{z}^{\prime}, \mathbf{T}\right) & =R_{T}\left(\omega_{0}, \boldsymbol{z}, \boldsymbol{z}^{\prime}, \mathbf{T}\right) \delta\left(\boldsymbol{z}-\boldsymbol{z}^{\prime}\right) .
\end{aligned}
$$

Note that $R_{\rho}$ is the average power of the electromagnetic radiation emitted by the scene at location $\boldsymbol{z}$, and $R_{T}$ is the average power of the electromagnetic radiation emitted by the transmitter at location $\mathbf{T}$ that is incident on the target surface at $\boldsymbol{z}$. In this regard, $R_{\rho}$ is referred to as the scene radiance and $R_{T}$ is referred to as the transmitter irradiance. ${ }^{18}$

Substituting $s_{i}$ and $s_{j}$ into $c_{i j}$, under the assumption that $\rho$ and $J_{\text {tr }}$ are statistically independent, and using (6) and (7), we express the expectation of $c_{i j}$ as follows:

$$
\begin{aligned}
E\left[c_{i j}\left(\tau^{\prime}, \tau, \mu\right)\right]= & \frac{\omega_{0}^{4}}{(4 \pi)^{4}} \int \mathrm{e}^{-\mathrm{i} \omega_{0}\left(t+\tau^{\prime}-\left(\left|\boldsymbol{\gamma}_{i}\left(t+\tau^{\prime}\right)-\mathbf{z}\right|+|\mathbf{T}-\mathbf{z}|\right) / c_{0}\right)} \mathrm{e}^{\mathrm{i} \omega_{0}\left(\mu t+\tau-\left(\left|\boldsymbol{\gamma}_{j}(\mu t+\tau)-\mathbf{z}^{\prime}\right|+\left|\mathbf{T}-\mathbf{z}^{\prime}\right|\right) / c_{0}\right)} \\
& \times \frac{R_{T}\left(\omega_{0}, \boldsymbol{z}, \boldsymbol{z}^{\prime}, \mathbf{T}\right) A_{R_{i j}}\left(\omega_{0}, \boldsymbol{z}, \boldsymbol{z}^{\prime}, t, \tau^{\prime}, \tau, \mu\right)}{G_{i j}\left(\mathbf{z}, \mathbf{z}^{\prime}, t, \tau^{\prime}, \tau, \mu\right) B_{i j}\left(\boldsymbol{z}_{0}, t, \tau^{\prime}, \tau, \mu\right)} R_{\rho}(\boldsymbol{z}) \delta\left(\boldsymbol{z}-\boldsymbol{z}^{\prime}\right) d \boldsymbol{z} d \boldsymbol{z}^{\prime} \phi(t)|t| d t
\end{aligned}
$$

where $A_{R_{i j}}$ is the product of the receiver antenna beam patterns,

$$
\left.\left.A_{R_{i j}}\left(\omega_{0}, \boldsymbol{z}, \boldsymbol{z}^{\prime}, t, \tau^{\prime}, \tau, \mu\right)=J_{\mathrm{rc}}\left(\omega_{0}, \mathbf{z}-\widehat{\gamma_{i}(t+} \tau^{\prime}\right), \gamma_{i}\left(t+\tau^{\prime}\right)\right) J_{\mathrm{rc}}^{*}\left(\omega_{0}, \mathbf{z}^{\prime}-\widehat{\gamma_{j}(\mu t}+\tau\right), \gamma_{j}(\mu t+\tau)\right)
$$

and $G_{i j}$ is the product of the geometric spreading factors,

$$
G_{i j}\left(\mathbf{z}, \mathbf{z}^{\prime}, t, \tau^{\prime}, \tau, \mu\right)=|\mathbf{T}-\mathbf{z}|\left|\mathbf{T}-\mathbf{z}^{\prime}\right|\left|\gamma_{i}\left(t+\tau^{\prime}\right)-\mathbf{z}\right|\left|\gamma_{j}(\mu t+\tau)-\mathbf{z}^{\prime}\right| .
$$

Note that for non-cooperative sources of opportunity, $\mathbf{T}$ and thus $|\mathbf{T}-\mathbf{z}|\left|\mathbf{T}-\mathbf{z}^{\prime}\right|$, are unknown.

Now using the Taylor series expansion of $\gamma_{i}\left(t+\tau^{\prime}\right)$ and $\gamma_{j}(\mu t+\tau)$ at $t=0$, we approximate

$$
\left|\gamma_{i}\left(t+\tau^{\prime}\right)-\mathbf{z}\right| \approx\left|\gamma_{i}\left(\tau^{\prime}\right)-\mathbf{z}+\dot{\gamma}_{i}\left(\tau^{\prime}\right) t\right| \approx\left|\gamma_{i}\left(\tau^{\prime}\right)-\mathbf{z}\right|+\left(\gamma_{i} \widehat{\left(\tau^{\prime}\right)}-\mathbf{z}\right) \cdot \dot{\gamma}_{i}\left(\tau^{\prime}\right) t
$$

and

$$
\left|\gamma_{j}(\mu t+\tau)-\mathbf{z}^{\prime}\right| \approx\left|\gamma_{j}(\tau)-\mathbf{z}^{\prime}+\dot{\gamma}_{j}(\tau) \mu t\right| \approx\left|\gamma_{j}(\tau)-\mathbf{z}^{\prime}\right|+\left(\gamma_{j} \widehat{(\tau)-} \mathbf{z}^{\prime}\right) \cdot \dot{\gamma}_{j}(\tau) \mu t
$$


Substituting the approximations back into (8), and performing the $\boldsymbol{z}^{\prime}$ integration, we have

$$
E\left[c_{i j}\left(\tau^{\prime}, \tau, \mu\right)\right] \approx \mathcal{F}_{i j}\left[R_{\rho}\right](\tau, \mu)=\int \mathrm{e}^{-\mathrm{i} \varphi_{i j}\left(t, \boldsymbol{z}, \tau^{\prime}, \tau, \mu\right)} \frac{A_{i j}\left(\boldsymbol{z}, t, \tau^{\prime}, \tau, \mu\right)}{B_{i j}\left(\boldsymbol{z}_{0}, t, \tau^{\prime}, \tau, \mu\right)} R_{\rho}(\boldsymbol{z}) d \boldsymbol{z}|t| d t
$$

where

$$
\varphi_{i j}\left(t, \boldsymbol{z}, \tau^{\prime}, \tau, \mu\right)=\omega_{0} t\left[1-\left(\widehat{\gamma_{j}(\tau)-} \mathbf{z}\right) \cdot \dot{\gamma}_{j}(\tau) / c_{0}\right]\left[S_{i j}\left(\tau^{\prime}, \tau, \mathbf{z}\right)-\mu\right]
$$

with

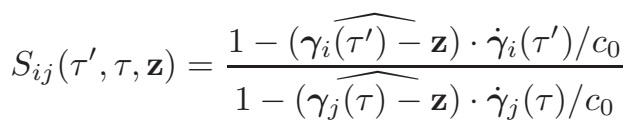

and

$$
A_{i j}\left(\boldsymbol{z}, t, \tau^{\prime}, \tau, \mu\right)=\frac{\omega_{0}^{4} \tilde{R}_{T}\left(\omega_{0}, \boldsymbol{z}\right) A_{R_{i j}}\left(\omega_{0}, \boldsymbol{z}, \boldsymbol{z}, t, \tau^{\prime}, \tau, \mu\right) \phi(t)}{(4 \pi)^{4} G_{i j}\left(\mathbf{z}, \mathbf{z}, t, \tau^{\prime}, \tau, \mu\right)} \mathrm{e}^{-\mathrm{i} \omega_{0}\left(\tau^{\prime}-\tau-\left(\left|\boldsymbol{\gamma}_{i}\left(\tau^{\prime}\right)-\mathbf{z}\right|-\left|\gamma_{j}(\tau)-\mathbf{z}\right|\right) / c_{0}\right)}
$$

with $\tilde{R}_{T}\left(\omega_{0}, \boldsymbol{z}\right)=R_{T}\left(\omega_{0}, \boldsymbol{z}, \boldsymbol{z}, \mathbf{T}\right)$. We refer to $S_{i j}\left(\tau^{\prime}, \tau, \mathbf{z}\right)$ as the Doppler-hitchhiker-scale-factor, and $\varphi_{i j}$ and $A_{i j} / B_{i j}$ as the phase and amplitude terms of the linear operator $\mathcal{F}_{i j}$.

For cooperative sources of opportunity, the transmitter locations and antenna beam patterns are known. Thus, $J_{\text {tr }}$ can be treated deterministically. $\tilde{R}_{T}\left(\omega_{0}, \boldsymbol{z}\right)$ in $(16)$ is replaced with $J_{\mathrm{tr}}\left(\omega_{0}, \widehat{\mathbf{z}-\mathbf{T}}, \mathbf{T}\right) J_{\mathrm{tr}}^{*}\left(\omega_{0}, \widehat{\mathbf{z}^{\prime}-\mathbf{T}}, \mathbf{T}\right)$.

Note that the filtered-scaled-and-translated correlation of the received signal removes all transmitter related terms from the phase of the operator $\mathcal{F}_{i j}$.

We assume that for some $m_{A}, A_{i j} / B_{i j}$ satisfies the following inequality:

$$
\sup _{(t, \mu, \tau, \boldsymbol{z}) \in \mathcal{U}}\left|\partial_{t}^{\alpha_{t}} \partial_{\mu}^{\alpha_{\mu}} \partial_{\tau}^{\beta} \partial_{z_{1}}^{\epsilon_{1}} \partial_{z_{2}}^{\epsilon_{2}} \frac{A_{i j}\left(\boldsymbol{z}, t, \tau^{\prime}, \tau, \mu\right)}{B_{i j}\left(\boldsymbol{z}_{0}, t, \tau^{\prime}, \tau, \mu\right)}\right| t|| \leq C_{A}\left(1+t^{2}\right)^{\left(m_{A}-\left|\alpha_{t}\right|\right) / 2}
$$

where $\mathcal{U}$ is any compact subset of $\mathbb{R}^{+} \times \mathbb{R}^{+} \times \mathbb{R} \times \mathbb{R} \times \mathbb{R}^{2}$, and the constant $C_{A}$ depends on $\mathcal{U}, \alpha_{t, \mu}, \beta, \epsilon_{1,2}$. This assumption is needed in order to make various stationary phase calculations hold. Under the assumption (17), (13) defines $\mathcal{F}$ as a Fourier integral operator whose leading order contributions come from those points lying in the intersection of the illuminated surface $(\boldsymbol{z}, \boldsymbol{\psi}(\boldsymbol{z}))$ and points that have the same Doppler-hitchhiker-scale-factor, i.e., $\left\{\mathbf{z} \in \mathbb{R}^{3}: S_{i j}\left(\tau^{\prime}, \tau, \mathbf{z}\right)=\mu\right\}$. We denote the curves formed by this intersection by

$$
F_{i j}\left(\tau^{\prime}, \tau, \mu\right)=\left\{\boldsymbol{z}: S_{i j}\left(\tau^{\prime}, \tau, \mathbf{z}=(\boldsymbol{z}, \psi(\boldsymbol{z}))\right)=\mu\right\} .
$$

When the speed of the receivers is much slower than the speed of light $c_{0}, S_{i j}\left(\tau^{\prime}, \tau, \mathbf{z}\right)=\mu$ defines a hitchhiker Doppler ${ }^{13}$ for a fixed frequency $\omega_{0}$. In this regard, we refer to $F_{i j}\left(\tau^{\prime}, \tau, \mu\right)$ as the DSAH iso-Doppler contour. Fig.2 shows the DSAH iso-Doppler contours for two receivers traversing a circular trajectory over a flat topography.

\section{IMAGE FORMATION}

We form an image of the scene radiance by the superposition of the weighted and backprojected data, $E\left[c_{i j}\left(\tau^{\prime}, \tau, \mu\right)\right]$ as follows:

$$
\tilde{R}_{\rho}(\boldsymbol{z})=\sum_{i j} \int \mathcal{K}_{i j}\left[E\left[c_{i j}\right]\right]\left(\boldsymbol{z}, \tau^{\prime}\right) d \tau^{\prime}
$$

where

$$
\mathcal{K}_{i j}\left[E\left[c_{i j}\right]\right]\left(\boldsymbol{z}, \tau^{\prime}\right)=\int \mathrm{e}^{\mathrm{i} \varphi_{i j}\left(t, \boldsymbol{z}, \tau^{\prime}, \tau, \mu\right)} Q_{i j}\left(\boldsymbol{z}, \tau^{\prime}, \tau, \mu\right) E\left[c_{i j}\left(\tau^{\prime}, \tau, \mu\right)\right] d t d \tau d \mu .
$$

We refer to $\mathcal{K}_{i j}$ as the weighted-backprojection operator with respect to the $i$ th and $j$ th receivers with weight $Q_{i j}$ to be determined below. 


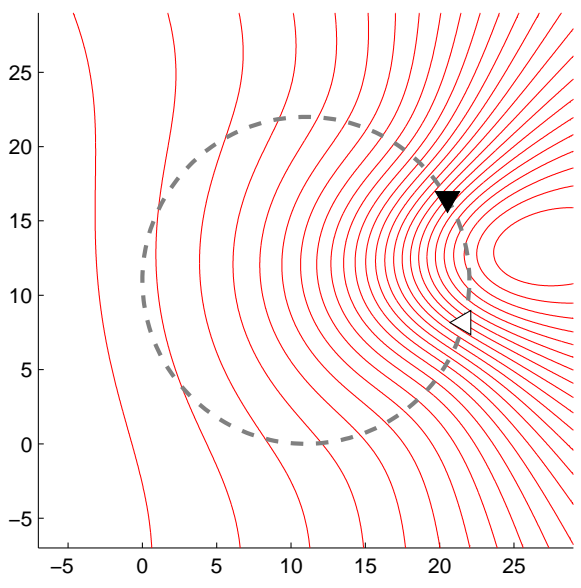

Figure 2: Iso-Doppler contours $F_{i j}\left(\tau^{\prime}, \tau, \mu\right)$ for the Doppler-hitchhiker-scale-factor $S_{12}(\pi / 6,-\pi / 12, \mathbf{z})$. with $\mu$ sampling over $[1-1.5 \mathrm{e}-6,1+1.5 \mathrm{e}-6]$. Two receivers are traversing a circular flight trajectory (dashed line) at the speed of $261 \mathrm{~m} / \mathrm{s}$ over a flat topography. The white and black triangles denote the positions of the two receivers.

We rewrite $\tilde{R}_{\rho}$ as

$$
\tilde{R}_{\rho}(\boldsymbol{z})=\sum_{i j} \mathcal{K}_{i j} \mathcal{F}_{i j}\left[R_{\rho}\right](\boldsymbol{z})=\int L\left(\boldsymbol{z}, \boldsymbol{z}^{\prime}\right) R_{\rho}\left(\boldsymbol{z}^{\prime}\right) d \boldsymbol{z}^{\prime}
$$

where $L\left(\boldsymbol{z}, \boldsymbol{z}^{\prime}\right)$ is the point spread function (PSF) of the imaging operator given by

$$
L\left(\boldsymbol{z}, \boldsymbol{z}^{\prime}\right)=\sum_{i j} \int L_{i j}\left(\boldsymbol{z}, \boldsymbol{z}^{\prime}, \tau^{\prime}\right) d \tau^{\prime}
$$

and

$$
L_{i j}\left(\boldsymbol{z}, \boldsymbol{z}^{\prime}, \tau^{\prime}\right)=\int \mathrm{e}^{\mathrm{i}\left[\varphi_{i j}\left(t, \boldsymbol{z}, \tau^{\prime}, \tau, \mu\right)-\varphi_{i j}\left(t^{\prime}, \boldsymbol{z}^{\prime}, \tau^{\prime}, \tau, \mu\right)\right]} Q_{i j}\left(\boldsymbol{z}, \tau^{\prime}, \tau, \mu\right) \frac{A_{i j}\left(\boldsymbol{z}^{\prime}, t, \tau^{\prime}, \tau, \mu\right)}{B_{i j}\left(\boldsymbol{z}, t, \tau^{\prime}, \tau, \mu\right)}|t| d t^{\prime} d t d \tau d \mu .
$$

Using the stationary phase theorem ${ }^{19}$ to approximate the $t^{\prime}$ and $\mu$ integrations, we obtain

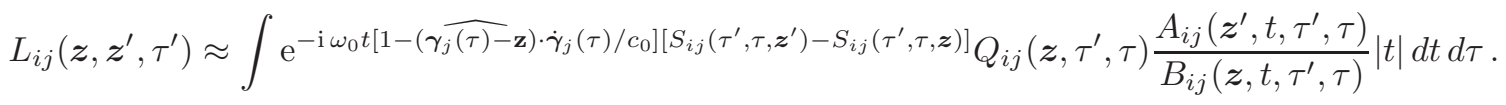

Applying the method of stationary phase to the $t$ and $\tau$ integrals, we see that the main contribution to $L_{i j}\left(\boldsymbol{z}, \boldsymbol{z}^{\prime}, \tau^{\prime}\right)$ comes from those critical points of its phase that satisfy the conditions:

$$
S_{i j}\left(\tau^{\prime}, \tau, \boldsymbol{z}^{\prime}\right)=S_{i j}\left(\tau^{\prime}, \tau, \boldsymbol{z}\right),
$$

and

$$
a_{j}^{\sum}(\tau, \mathbf{z}) /\left[1-\left(\widehat{\gamma_{j}(\tau)-} \mathbf{z}\right) \cdot \dot{\gamma}_{j}(\tau) / c_{0}\right]=a_{j}^{\sum}\left(\tau, \mathbf{z}^{\prime}\right) /\left[1-\left(\gamma_{j} \widehat{(\tau)-} \mathbf{z}^{\prime}\right) \cdot \dot{\gamma}_{j}(\tau) / c_{0}\right]
$$

where

$$
a_{j}^{\sum_{j}}(\tau, \mathbf{z})=\frac{1}{\left|\gamma_{j}(\tau)-\mathbf{z}\right|}\left[\dot{\gamma}_{j, \perp}(\tau, \mathbf{z}) \cdot \dot{\gamma}_{j}(\tau)\right]^{2}+\left(\gamma_{j} \widehat{(\tau)-} \mathbf{z}\right) \cdot \ddot{\gamma}_{j}(\tau)
$$




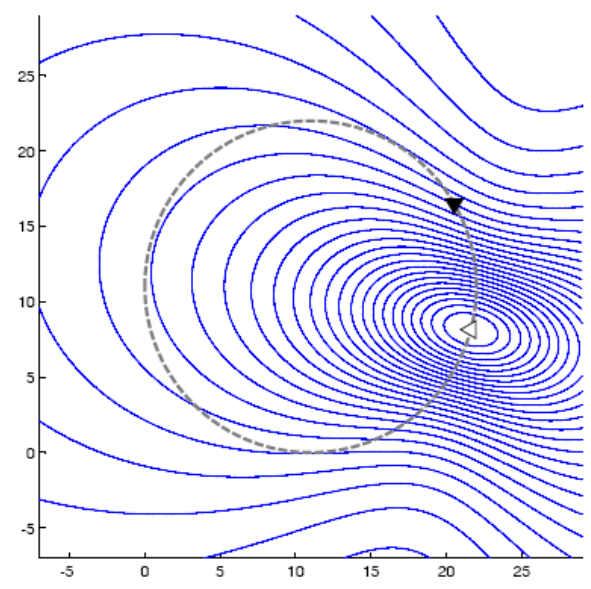

Figure 3: Iso-Doppler-rate contours $\dot{F}_{j}(\tau, C)$ for the DSAH Doppler-rate $f_{1}(-\pi / 12, \mathbf{z})$. Two receivers are traversing a circular flight trajectory (dashed line) at the speed of $261 \mathrm{~m} / \mathrm{s}$ over a flat topography. The white and black triangles denote the positions of the two receivers .

Note that $\dot{\gamma}_{j, \perp}(\tau, \mathbf{z})$ is the projection of the receiver velocity $\dot{\gamma}_{j}(\tau)$ onto the plane whose normal direction is along $\widehat{\gamma_{j}(\tau)-} \mathbf{z}$ and $a_{j}^{\sum}(\tau, \mathbf{z})$ is the total relative radial acceleration of the $j$ th receiver in the direction of $\boldsymbol{\gamma}_{j} \widehat{(\tau)-} \mathbf{z}$.

We define

$$
f_{j}(\tau, \mathbf{z}):=a_{j}^{\sum}(\tau, \mathbf{z}) /\left[1-\left(\gamma_{j} \widehat{(\tau)-} \mathbf{z}\right) \cdot \dot{\gamma}_{j}(\tau) / c_{0}\right]
$$

and refer to $f_{j}(\tau, \mathbf{z})$ as the DSAH Doppler-rate of the $j$ th receiver. We refer to the locus of points that is formed by the intersection of the surface topography and $\left\{\mathbf{z} \in \mathbb{R}^{3}: f_{j}(\tau, \mathbf{z})=C\right\}$, for some constant $C$, as the DSAH iso-Doppler-rate contour, and denote it by $\dot{F}_{j}(\tau, C)=\left\{\boldsymbol{z}: f_{j}(\tau, \boldsymbol{z})=C\right\}$. Figure 3 shows the DSAH iso-Doppler-rate contours with fixed $\tau$ for a circular flight trajectory and flat topography.

The critical points $\boldsymbol{z}$ of the phase of $L_{i j}\left(\boldsymbol{z}, \boldsymbol{z}^{\prime}, \tau^{\prime}\right)$ are those points lying on the intersection of the DSAH iso-Doppler curves $F_{i j}\left(\tau^{\prime}, \tau, \mu\right)$ and DSAH iso-Doppler-rate curves $\dot{F}_{j}(\tau, C)$. We assume that the only critical point within the region of interest is $\boldsymbol{z}=\boldsymbol{z}^{\prime}$.

To determine the weight and the filter, we linearize $S_{i j}\left(\tau^{\prime}, \tau, \boldsymbol{z}^{\prime}\right)$ around $\boldsymbol{z}^{\prime}=\boldsymbol{z}$ and approximate

$$
S_{i j}\left(\tau^{\prime}, \tau, \boldsymbol{z}^{\prime}\right)-S_{i j}\left(\tau^{\prime}, \tau, \boldsymbol{z}\right) \approx \nabla_{\boldsymbol{z}} S_{i j}\left(\tau^{\prime}, \tau, \boldsymbol{z}\right) \cdot\left(\boldsymbol{z}^{\prime}-\boldsymbol{z}\right)
$$

and

$$
A_{i j}\left(\boldsymbol{z}^{\prime}, t, \tau^{\prime}, \tau\right) \approx A_{i j}\left(\boldsymbol{z}, t, \tau^{\prime}, \tau\right)
$$

to write

$$
L_{i j}\left(\boldsymbol{z}, \boldsymbol{z}^{\prime}, \tau^{\prime}\right)=\int \mathrm{e}^{-\mathrm{i} t \boldsymbol{\Xi}_{i j}\left(\tau^{\prime}, \tau, \boldsymbol{z}\right) \cdot\left(\boldsymbol{z}^{\prime}-\boldsymbol{z}\right)} Q_{i j}\left(\boldsymbol{z}, \tau^{\prime}, \tau\right) \frac{A_{i j}\left(\boldsymbol{z}, t, \tau^{\prime}, \tau\right)}{B_{i j}\left(\boldsymbol{z}, t, \tau^{\prime}, \tau\right)}|t| d t d \tau
$$

where

$$
\boldsymbol{\Xi}_{i j}\left(\tau^{\prime}, \tau, \boldsymbol{z}\right)=\omega_{0}\left[1-\left(\gamma_{j} \widehat{(\tau)-} \mathbf{z}\right) \cdot \dot{\gamma}_{j}(\tau) / c_{0}\right] \nabla_{\boldsymbol{z}} S_{i j}\left(\tau^{\prime}, \tau, \boldsymbol{z}\right) .
$$

In (30) for each $\tau^{\prime}$ and $\boldsymbol{z}$, we make the following change of variables:

$$
(t, \tau) \rightarrow \boldsymbol{\xi}_{i j}=t \boldsymbol{\Xi}_{i j}\left(\tau^{\prime}, \tau, \boldsymbol{z}\right)
$$

and we choose the weight and the filter as follows:

$$
Q_{i j}\left(\boldsymbol{z}, \tau^{\prime}, \tau\right)=\left[|t|,\left|\frac{\partial(t, \tau)}{\partial \boldsymbol{\xi}_{i j}}\right|\right]^{-1}=\left|\operatorname{det}\left[\begin{array}{c}
\boldsymbol{\Xi}_{i j}\left(\tau^{\prime}, \tau, \boldsymbol{z}\right) \\
\partial_{\tau} \boldsymbol{\Xi}_{i j}\left(\tau^{\prime}, \tau, \boldsymbol{z}\right)
\end{array}\right]\right|
$$


and

$$
B_{i j}^{-1}\left(\boldsymbol{z}, t, \tau^{\prime}, \tau, \mu\right)=A_{i j}^{-1}\left(\boldsymbol{z}, t, \tau^{\prime}, \tau, \mu\right) \chi_{\Omega_{i j, \tau^{\prime}, \boldsymbol{z}}}\left(\boldsymbol{z}, t, \tau^{\prime}, \tau\right)
$$

where $\chi_{\Omega_{i j, \tau^{\prime}, z}}$ is a smooth cut-off function equal to one in the interior of $\Omega_{i j, \tau^{\prime}, \boldsymbol{z}}$ and zero in the exterior of $\Omega_{i j, \tau^{\prime}, \boldsymbol{z}}$ with $\Omega_{i j, \tau^{\prime}, \boldsymbol{z}}$ given by

$$
\Omega_{i j, \tau^{\prime}, \boldsymbol{z}}=\left\{\boldsymbol{\xi}_{i j}=t \boldsymbol{\Xi}_{i j}\left(\tau^{\prime}, \tau, \boldsymbol{z}\right) \mid A_{i j}\left(\boldsymbol{z}, t, \tau^{\prime}, \tau\right) \neq 0,\left(t, \tau^{\prime}, \tau\right) \in\left(\mathbb{R}^{+}, \mathbb{R}, \mathbb{R}\right)\right\} .
$$

We refer to $\Omega_{i j, \tau^{\prime}, \boldsymbol{z}}$ as the partial data collection manifold at $\left(\tau^{\prime}, \boldsymbol{z}\right)$ obtained by the $i$ th and $j$ th receivers for a fixed $\tau^{\prime}$ and refer to the union $\cup_{i j, \tau^{\prime}} \Omega_{i j, \tau^{\prime}, \boldsymbol{z}}$ as the data collection manifold at $\boldsymbol{z}$ and denote it by $\Omega_{\boldsymbol{z}}$. This set determines many of the properties of the image.

To avoid instability of $(33), B_{i j}\left(\boldsymbol{z}, t, \tau^{\prime}, \tau, \mu\right)$ can be modified to

$$
B_{i j}^{-1}\left(\boldsymbol{z}, t, \tau^{\prime}, \tau, \mu\right)=\frac{\overline{A_{i j}\left(\boldsymbol{z}, t, \tau^{\prime}, \tau, \mu\right)} \chi_{\Omega_{i j, \tau^{\prime}, \boldsymbol{z}}}\left(\boldsymbol{z}, t, \tau^{\prime}, \tau\right)}{\left|A_{i j}\left(\boldsymbol{z}, t, \tau^{\prime}, \tau, \mu\right)\right|^{2}+\sigma^{2}}
$$

where $\sigma^{2}$ is a small positive constant.

Note that we choose the filter to compensate for the terms involving antenna beam patterns and geometric spreading functions and the weight to perform proper interpolation in the phase space going from $(t, \tau)$ to $\boldsymbol{\xi}_{i j}$ coordinates. These choices make the leading order term of $L_{i j}\left(\boldsymbol{z}, \boldsymbol{z}^{\prime}, \tau^{\prime}\right)$ in (30) to be the Dirac-delta function.

Substituting (31), (32) and (33) into (20), we obtain

$$
\tilde{R}_{\rho}(\boldsymbol{z})=\sum_{i j} \mathcal{K}_{i j}\left[\mathcal{F}_{i j}\left[R_{\rho}\right]\right](\boldsymbol{z}) \approx \sum_{i j} \int_{\Omega_{i j, \tau^{\prime}, \boldsymbol{z}}} \mathrm{e}^{-\mathrm{i} \boldsymbol{\xi}_{i j} \cdot\left(\boldsymbol{z}^{\prime}-\boldsymbol{z}\right)} R_{\rho}\left(\boldsymbol{z}^{\prime}\right) d \boldsymbol{z}^{\prime} d \boldsymbol{\xi}_{i j} d \tau^{\prime} .
$$

(36) shows that the image $\tilde{R}_{\rho}$ is a band-limited version of $R_{\rho}$ whose band-width is determined by the data collection manifold $\Omega_{\boldsymbol{z}}$, which describes the resolution of the reconstructed image $\tilde{R}_{\rho}$ at $\boldsymbol{z}$. The larger the data collection manifold, the better the resolution of the image is.

Microlocal analysis of (36) tell us that an edge at point $\boldsymbol{z}$ is visible if the direction $\boldsymbol{n}_{\boldsymbol{z}}$ normal to the edge is contained in $\Omega_{\boldsymbol{z}}{ }^{14}$ Consequently, an edge at point $\boldsymbol{z}$ with $\boldsymbol{n}_{\boldsymbol{z}}$ normal to edge is visible if there exists $i, j, \tau^{\prime}, \tau$ such that $\boldsymbol{\xi}_{i j}$ is parallel to $\boldsymbol{n}_{\boldsymbol{z}}$. Furthermore, the band-width contribution of $\boldsymbol{\xi}_{i j}=t \boldsymbol{\Xi}_{i j}\left(\tau^{\prime}, \tau, \boldsymbol{z}\right)$ to a visible edge at $\boldsymbol{z}$ is given by $L_{\phi}\left|\boldsymbol{\Xi}_{i j}\left(\tau^{\prime}, \tau, \boldsymbol{z}\right)\right|$ where $L_{\phi}$ denotes the length of the support of $\phi(t)$. Thus, longer the support of $\phi(t)$, larger the magnitude of $\boldsymbol{\xi}_{i j}$ becomes, giving rise to sharper reconstructed edges perpendicular to $\boldsymbol{\xi}_{i j}$, $i, j=1, \ldots, N$. Additionally, higher the $\omega_{0}$, the frequency of the transmitted signal, larger the magnitude of $\boldsymbol{\xi}_{i j}$ becomes, contributing to higher image resolution.

(36) shows that irrespective of the choice of the weight and filter, the backprojection operator recovers the visible edges of the scene radiance at the right location for a given $i$ th and $j$ th receivers and a fixed $\tau^{\prime}$. With the choice of the weight and filter given in (32) and (33), respectively, the resulting image formation algorithm recovers the visible edges of the scene radiance not only at the right location and orientation, but also at the right strength.

\section{NUMERICAL SIMULATIONS}

We conducted numerical simulations to demonstrate the performance of our imaging method using a multiplepoint-target model. We considered a scene of size $[0,22] \times[0,22] \mathrm{km}^{2}$ with flat topography. The scene was discretized by $128 \times 128$ pixels, where $[0,0,0] \mathrm{km}$ and $[22,22,0] \mathrm{km}$ correspond to the pixels $(1,1)$ and $(128,128)$, respectively.

In all the numerical experiments, we used two airborne receivers and a single, stationary transmitter operating either cooperatively or non-cooperatively. We assumed that both the receiver and transmitter antennas were 


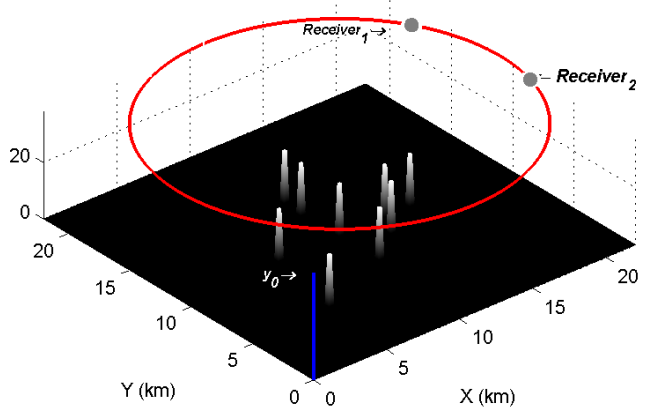

(a)

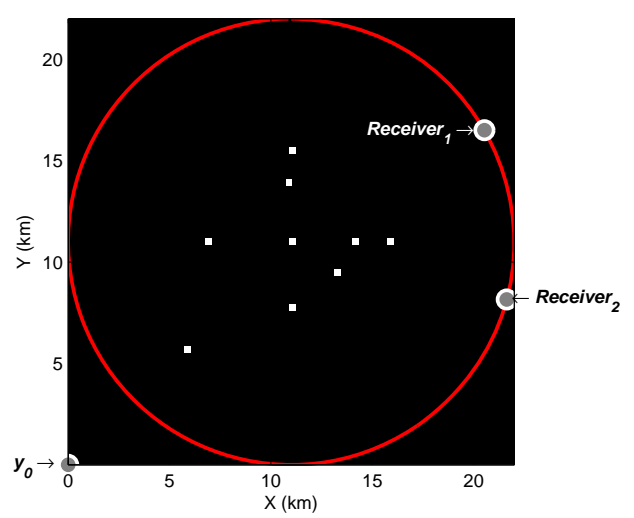

(b)

Figure 4: (a) 3-D and (b) 2-D views of the scene with multiple point targets, illuminated by a single transmitter located at $\mathbf{y}_{0}=(0,0,6.5) \mathrm{km}$ and the circular receiver trajectory $\gamma_{C}(s)=(11+11 \cos (s), 11+11 \sin (s), 6.5) \mathrm{km}$, as shown by the red solid line. At a certain time instant, two receivers are located at the positions shown in the figure.

isotropic. We assumed that the transmitter was located at $\mathbf{y}_{0}=(0,0,6.5) \mathrm{km}$ and the receivers were traversing the circular trajectory given by

$$
\gamma_{C}(s)=(11+11 \cos (s), 11+11 \sin (s), 6.5) \mathrm{km} .
$$

Let $\gamma_{1}(s)$ and $\gamma_{1}(s)$ denote the trajectories of the two receivers. We set $\gamma_{1}(s)=\gamma_{C}(s)$ and $\gamma_{2}(s)=\gamma_{1}\left(s-\frac{\pi}{6}\right)$. Note that the variable $s$ in $\gamma_{C}$ is equal to $\frac{V}{R} t$ where $V$ is the speed of the receiver, and $R$ is the radius of the circular trajectory. We set the speed of the two receivers to $261 \mathrm{~m} / \mathrm{s}$. We chose the sampling rate of $\tau$ to be $1.9335 \mathrm{~Hz}$ so as to uniformly sample the circular trajectory with 512 points.

For all the numerical experiments, we used (1) to generate the data and chose the windowing function $\phi$ in (3) to be a Hanning function. We performed image reconstruction for each $\tau^{\prime}$ and coherently superimposed the reconstructed images obtained over a range of $\tau^{\prime}$.

In accordance with the incoherent field approximation, we used the following multiple-point-target model for the scene reflectivity

$$
\rho(\boldsymbol{z})=\sum_{l=1}^{L} g_{l} \delta\left(\boldsymbol{z}-\boldsymbol{z}_{l}\right)
$$

where $g_{l}, l=1, \cdots, L$ are independent Gaussian random variables with mean $\mu_{l}$ and variance $\sigma_{l}^{2}$. The corresponding scene radiance is given by

$$
R_{\rho}(\boldsymbol{z})=E\left[\rho(\boldsymbol{z}) \rho^{*}(\boldsymbol{z})\right]=\sum_{l}\left(\mu_{l}^{2}+\sigma_{l}^{2}\right) \sigma\left(\boldsymbol{z}-\boldsymbol{z}_{l}\right) .
$$

In our simulations, we considered a deterministic reflectivity and set $\sigma_{l}^{2}=1$. We used $L=9$ and approximated the Dirac-delta functions in (39) by square target reflectors of size $344 \times 344 \mathrm{~m}^{2}$, each having a unit reflectivity, i.e., $\mu_{l}=1, l=1, \cdots, 9$. The Figs.4(a) and $4(\mathrm{~b})$ show the scene with targets, receiver trajectories and the transmitter antenna location.

\subsection{Cooperative Transmitters -}

For the case of a cooperative transmitter, the reconstructed image is shown in Fig.5. The two images corresponding to the fixed $\tau^{\prime}$ values are shown in Fig.6(a) and Fig.6(b). As expected, due to a smaller data collection 


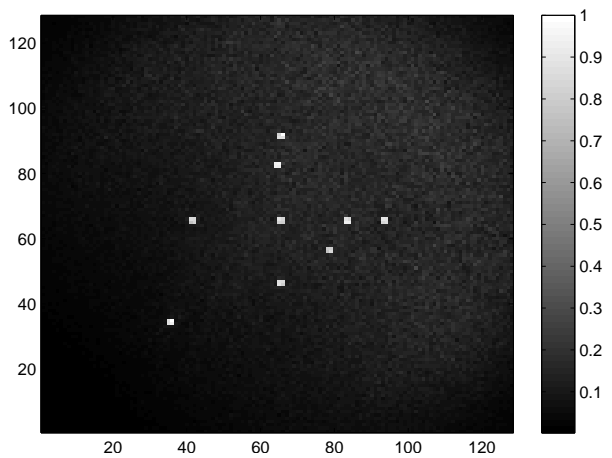

Figure 5: The reconstructed image for multiple point targets with $16 \tau^{\prime}$ values uniformly spaced in [0, 248.258]s using a single cooperative transmitter located at $\mathbf{y}_{0}$ and two receivers traversing the circular flight trajectories $\gamma_{1}(s)$ and $\gamma_{2}(s)$ as shown in Fig.4.

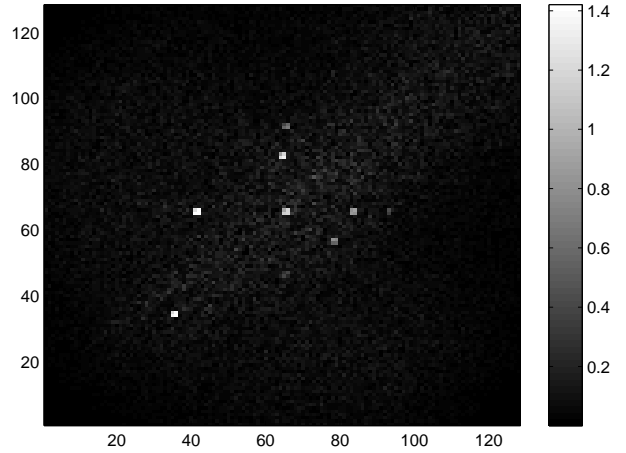

(a)

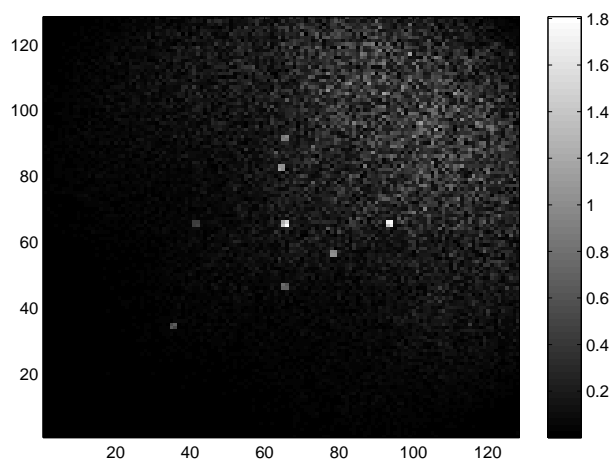

(b)

Figure 6: The images reconstructed for multiple point targets using the vectors in the partial data collection manifolds (a) $\Omega_{12, \tau^{\prime}=33.1011 \mathrm{~s}}$ (corresponding to $s=\pi / 4$ ), and (b) $\Omega_{12, \tau^{\prime}=165.5054 \mathrm{~s}}$ (corresponding to $s=1.25 \pi$ ), with a single cooperative transmitter located at $\mathbf{y}_{0}$ and two receivers traversing circular flight trajectories $\gamma_{1}(s)$ and $\gamma_{2}(s)$ as shown in Fig.4.

manifold, the resolution of these images is poorer than that of the image obtained by superposition of all the images reconstructed for a range of $\tau^{\prime}$ values, $\tau^{\prime} \in[0,248.258] \mathrm{s}$, as shown in Fig.5. We observe that some of the targets do not appear as sharp as the ones in Fig.5.

\subsection{Non-cooperative Transmitters -}

Fig.7 shows the reconstructed image for multiple point targets using a non-cooperative transmitter. Since the location of the transmitter was assumed to be unknown, the received signal was not compensated for the transmitter related geometric spreading factors. As a result, the scatterers closer to the transmitter appears brighter in the reconstructed image than those that are further away from the transmitter.

\section{CONCLUSION}

In this paper, we developed a novel image formation method for passive SAR that uses transmitters of opportunity with fixed-frequency waveforms and receivers traversing arbitrary flight trajectories. The method is based on the windowed, filtered-scaled-and-translated correlation of the received signals at different (or the same) receiver and weighted-backprojection of the resulting correlated signal. Such preprocessing of the received signal removes the transmitter related terms from the phase of the resulting forward model allowing us to perform backprojection without having the knowledge of transmitter locations. 


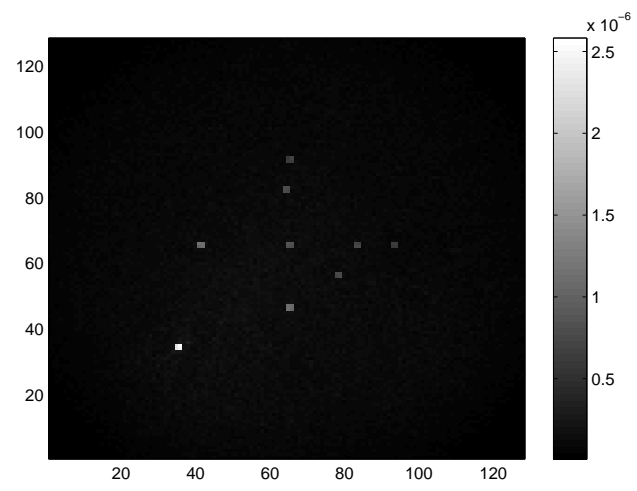

Figure 7: The reconstructed image for multiple point targets with $16 \tau^{\prime}$ values uniformly sampled in $[0,248.258] \mathrm{s}$ using a single non-cooperative transmitter located at $\mathbf{y}_{0}$ and two receivers traversing the circular flight trajectories $\gamma_{1}(s)$ and $\gamma_{2}(s)$ as shown in Fig.4.

The windowed, filtered-scaled-and-translated correlations of the received signal results in the projection of the ground radiance onto the curves defined by the Doppler-hitchhiker-scale-factor. We next used microlocal analysis to recover ground radiance from its projections via weighted-backprojection. The analysis of the point spread function of the imaging operator shows that the singularities of the scene radiance that are reconstructed are those that are at the intersection of the curves defined by the Doppler-hitchhiker-scale-factor and hitchhiker iso-Doppler-rate curves. The weighted-backprojection algorithm puts the visible edges of the scene radiance at the correct location, and under appropriate conditions, with correct strength. The analysis of the Fourier space data collection manifold shows that the resolution of the reconstructed image depends on the frequency of the transmitter, the length of the support of the windowing function, the hitchhiker Doppler and the range of the $\tau^{\prime}$ variable. The numerical simulations demonstrate the performance of the reconstruction algorithm.

We described our imaging algorithm for a single, stationary transmitter. However, the method can be easily extended to multiple mobile or stationary transmitters by simply integrating the received signal model over all transmitter locations similar to our work in. ${ }^{13}$ The method can be extended to include the case where the received data is contaminated with additive noise following the framework introduced in our prior work. ${ }^{17}$

Although we presented our approach specifically for passive synthetic aperture radar, our method is also applicable to other passive imaging problems, such as passive geophysical or acoustic imaging.

\section{ACKNOWLEDGMENTS}

This work is supported by the Air Force Office of Scientific Research (AFOSR) under the agreement FA9550-071-0363 and by the National Science Foundation (NSF) under Grant No. CCF-08030672.

\section{REFERENCES}

[1] Griffiths, H. D. and Long, N. R. W., "Television-based bistatic radar," IEE Proc.-Radar Sonar Navig. 133(7), 649-657 (1986).

[2] Homer, J., Kubik, K., Mojarrabi, B., Longstaff, I., Donskoi, E., and Cherniakov, M., "Passive bistatic radar sensing with leos based transmitters," in [Proceedings of IEEE International Geoscience and Remote Sensing Symposium], 1, 438-440 (June 2002).

[3] Griffiths, H. D. and Baker, C. J., "Passive coherent location radar systems. part 1: Performance prediction," IEE Proc.-Radar Sonar Navig. 152 (June 2005).

[4] Poullin, D., "Passive detection using digital broadcasters (DAB, DVB) with COFDM modulation," IEE Proc.-Radar Sonar Navig. 152, 143-152 (June 2005).

[5] Koch, V. and Westphal, R., "New approach to a multistatic passive radar sensor for air/space defense," IEEE Aerospace and Electronic Systems Magazine 10, 24-32 (Nov. 1995). 
[6] Cherniakov, M., Kubik, K., and Nezlin, D., "Bistatic synthetic aperture radar with non-cooperative leos based transmitter," in [Proceedings of IEEE International Geoscience and Remote Sensing Symposium], 2, 861-862 (July 2000).

[7] Cherniakov, M., Kubik, K., and Nezlin, D., "Radar sensors based on communication low earth orbiting satellites microwave emission," in [Proceedings of IEEE International Geoscience and Remote Sensing Symposium], 3, 1007-1008 (July 2000).

[8] Howland, P. E., Maksimiuk, D., and Reitsma, G., "Fm radio based bistatic radar," IEE Proceeings - Radar, Sonar and Navigation 152, 107-115 (June 2005).

[9] Baker, C. J., Griffiths, H. D., and Papoutsis, I., "Passive coherent location radar systems. part 2: Waveform properties," IEE Proc.-Radar Sonar Navig. 152, 160-168 (June 2005).

[10] Tan, D., Sun, H., Lu, Y., Lesturgie, M., and Chan, H. L., "Passive radar using global system for mobile communication signal: theory, implementation and measurements," IEE Proc.-Radar Sonar Navig. 152, 116-123 (June 2005).

[11] He, X., Cherniakov, M., and Zeng, T., "Signal detectability in ss-bsar with gnss non-cooperative transmitter," IEE Proc.-Radar Sonar Navig. 152, 124-132 (June 2005).

[12] Antoniou, M., Cherniakov, M., and Hu, C., "Space-surface bistatic sar image formation algorithms," Geoscience and Remote Sensing, IEEE Transactions on 47, 1827-1843 (June 2009).

[13] Yarman, C. and Yazıcı, B., "Synthetic aperture hitchhiker imaging," IEEE Transactions on Imaging Processing 17, 2156-2173 (Nov. 2008).

[14] Nolan, C. J. and Cheney, M., "Synthetic aperture inversion," Inverse Problems 18, 221-236 (2002).

[15] Nolan, C. and Cheney, M., "Synthetic aperture inversion for arbitrary flight paths and non-flat topography," IEEE Transactions on Image Processing 12, 1035-1043 (2003).

[16] Quinto, E., "Singularities of the x-ray transform and limited data tomography in $r^{2}$ and $r^{3}$," SIAM J. Math. Anal. 24, 1215-1225 (1993).

[17] Yazici, B., Cheney, M., and Yarman, C., "Synthetic-aperture inversion in the presence of noise and clutter," Inverse Problems 22, 1705-1729 (2006).

[18] Barrett, H. and Myers, K., [Foundations of image science], Wiley-Interscience, Hoboken, NJ (2004).

[19] Bleistein, N. and Handelsman, R., [Asymptotic Expansions of Integrals], Dover, New York (1986). 